\title{
Increase in Hypotonic Stress-Induced Endocytic Activity in Macrophages via CIC-3
}

\author{
Yutao Yan ${ }^{1,2}$, Yu Ding ${ }^{1}$, Bingxia Ming ${ }^{1}$, Wenjiao Du ${ }^{1}$, Xiaoling Kong ${ }^{1}$, Li Tian ${ }^{1}$, Fang Zheng ${ }^{1}$, \\ Min Fang ${ }^{1}$, Zheng Tan ${ }^{1, *}$, and Feili Gong ${ }^{1, *}$
}

\begin{abstract}
Extracellular hypotonic stress can affect cellular function. Whether and how hypotonicity affects immune cell function remains to be elucidated. Macrophages are immune cells that play key roles in adaptive and innate in immune reactions. The purpose of this study was to investigate the role and underlying mechanism of hypotonic stress in the function of bone marrow-derived macrophages (BMDMs). Hypotonic stress increased endocytic activity in BMDMs, but there was no significant change in the expression of CD80, CD86, and MHC class II molecules, nor in the secretion of TNF- $\alpha$ or IL-10 by BMDMs. Furthermore, the enhanced endocytic activity of BMDMs triggered by hypotonic stress was significantly inhibited by chloride channel-3 (CIC-3) siRNA. Our findings suggest that hypotonic stress can induce endocytosis in BMDMs and that $\mathrm{CIC}-3$ plays a central role in the endocytic process.
\end{abstract}

\section{INTRODUCTION}

Macrophages are multi-functional immune cells present in both peripheral blood and tissues (Cassetta et al., 2011). As innate immune cells, macrophages engulf infectious microorganisms, phagocytose tissue debris and apoptotic parenchymal cells, and repair wounded tissue (Shapiro et al., 2011). Macrophages are also involved in adaptive immunity by acting as antigenpresenting cells (APC) which initiate T cell-mediated immune responses (Medina-Contreras et al., 2011). Activated macrophages secrete a variety of cytokines which mediate immunity and inflammatory responses (Bozza et al., 2012; Liu et al., 2012). Macrophages are also associated with the occurrence and development of many diseases. For example, recent studies have shown a large influx of macrophages during myocardial infarction influence disease progression (Thorp, 2012).

\footnotetext{
${ }^{1}$ Department of Immunology, Tongji Medical College, ${ }^{2}$ Institute of Organ Transplantation, Tongji Hospital, Tongji Medical College, Huazhong University of Science and Technology, Wuhan 4340030, China
} *Correspondence: flgong@ 163.com (FG); tanzheng @ hust.edu.cn (ZT)

Received 13 February, 2014; accepted 1 April, 2014; published online 16 May, 2014

Keywords: chloride channels -3 , endocytosis, hypotonic stress, macrophage
Local tissue osmotic pressure plays a pivotal role in normal homeostatic mechanisms. Tissue osmotic pressure can be altered by hydration, as well as non-electrolyte and ion exchange. Vasogenic cerebral edema and cytotoxic cerebral edema resulting from bacterial meningitis are both characterized by hypotonicity (Tunkel and Scheld, 1993). Osmoregulation is the homeostatic mechanism of an organism, which maintains osmotic pressure stability (Schliess et al., 2004). Disruption of osmoregulation may lead to abnormal osmotic pressure and produce pathogenic consequences, for example, cirrhosis associated with hypotonic encephalopathy (Gaglio et al., 2012), acidosis and ischemia-reperfusion injury (Kajimoto et al., 2011).

The accumulation of intracellular metabolic products during an ischemic episode can affect local tissue osmotic pressure and alter intracellular ion concentrations, aggravating damage during reperfusion (Roberts and Christini, 2011). Rapid removal of intracellular metabolic products during reperfusion results in an extracellular hypotonic state in which extracellular water permeates the cells and causes cell swelling, rupture and necrosis. Strategies to control extracellular osmotic pressure have been used to treat diseases in the clinic (Dickerson et al., 2013; Siegel, 2007). Intraperitoneal chemotherapy with infusion of hypotonic fluids has been used for adjuvant post-surgical or palliative treatment of ovarian, colon, liver, and gastrointestinal malignancies (Kondo et al., 1996). However, the effect of hypotonic stress on macrophages has not been elucidated.

Most investigators believe that alteration of cellular function by a hypotonic microenvironment relies on the participation of chloride channels $\mathrm{CIC}-2$ and $\mathrm{CIC}-3$, members of the $\mathrm{CIC}$ voltage-gated chloride channel superfamily. Mutations in these channels produce several genetic diseases, including myotonia (CIC-1) (Wheeler et al., 2007), cardiovascular disease (CIC-3) (Duan, 2011), and hereditary nephrocalcinosis (Dent's disease), resulting from mutations in CIC-5 (Mohammad-Panah et al., 2003).

In this study, we investigated the effect of hypotonic stress on macrophage function and report that a hypotonic environment upregulates endocytic activity in macrophages via $\mathrm{CIC}-3$.

\section{MATERIALS AND METHODS}

\section{Main reagents}

The isotonic or hypotonic solution contained $(\mathrm{mM}): \mathrm{NaCl} 95$, $\mathrm{KCl} 4.5, \mathrm{CaCl}_{2} 1, \mathrm{MgCl}_{2} 1$, Hepes 5, D-mannitol 110 or $0(\mathrm{pH}$ 7.4, adjusted with $\mathrm{NaOH}, 310$ or $200 \mathrm{mOsm} / \mathrm{kg} \mathrm{H}_{2} \mathrm{O}$ ) 
(Abdullaev et al., 2003; Mohammad-Panah et al.). M-CSF was purchased from PeproTech (USA), and CD16/32 from Biolegend (USA). The fluorescent antobodies (Abs), including FITClabeled anti-CD80, PE-labeled anti-CD86, PE-cy5-labeled $\mathrm{MHC}$ class II, and APC-labeled F4/80 were bought from eBioscience (USA). Both TNF- $\alpha$ and IL-10 mouse ELISA kits were from Biolegend (USA). TRIzol reagent was from Invitrogen (USA). PrimeScript 1st Strand cDNA Synthesis Kit was from TransGen Biotech (China). Rabbit anti-CIC-3 or rabbit anti-CIC2 Abs were from Alomone Laboratories (Israel). Anti-GAPDH $\mathrm{Ab}$ was from BD Biosciences (USA). FITC-conjugated goat anti-rabbit secondary $A b$ was from eBioscience (USA).

\section{Cell culture}

Female C57BL/6 mice, 6-8 weeks old, were obtained from the Experimental Animal Center of Tongji Medical College, Huazhong University of Science and Technology (China). All mice were maintained under specific pathogen-free conditions and the studies were performed according to the guidelines of the Animal Care and Use Committee of Tongji Medical College, Huazhong University of Science and Technology (China).

BMDMs were generated as previously described with minor modifications (Gong et al., 2012; Park and Bryers, 2012). In brief, bone marrow cells were obtained from the femurs and tibias of C57BL/6 mice at a density of $1 \times 10^{6} / \mathrm{ml}$ in DMEMs medium supplemented with $10 \%$ heat-inactivated FBS, penicillin $(100 \mu \mathrm{g} / \mathrm{ml})$, streptomycin $(100 \mu \mathrm{g} / \mathrm{ml})$, then seeded in 6-well plates $(2 \mathrm{ml} /$ well). Cultures were added with $10 \mathrm{ng} / \mathrm{ml} \mathrm{M-CSF}$. The cells were incubated for 7 days, and the medium were changed at the day 3 and 5 by aspirating $75 \%$ of the medium and adding back fresh medium containing $10 \mathrm{ng} / \mathrm{ml}$ M-CSF. BMDMs were harvested by trypsinization, and used for experiments. The cells were characterized as closely adherent mononuclear cells which expressed high levels of F4/80.

The BMDMs were stimulated by isotonic or hypotonic solution for $15 \mathrm{~min}$, then cultured in normal environment $\left(37^{\circ} \mathrm{C}, 5 \%\right.$ $\mathrm{CO}_{2}$ ) for the following experiments.

\section{Apoptosis detection}

Apoptosis was determined by annexin V-FITC/PI staining using flow cytometry. BMDMs were pretreated with hypotonic solution or isotonic solution for 15 min respectively. Thereafter, cells at a density of $1 \times 10^{6}$ were collected, centrifuged and washed with PBS for two times. Binding buffer was then added to each tube and cells were re-suspended. The re-suspended cells were incubated with $5 \mu \mathrm{l}$ annexin V-FITC and $10 \mu \mathrm{l}$ of PI for $15 \mathrm{~min}$ at room temperature in the dark. Then, the percentage of apoptotic cells was determined by using flow cytometry. BMDMs which pretreated with isotonic served as control group.

\section{Pinocytosis and phagocytosis assay}

To measure the pinocytotic activity of BMDMs, a previously reported method was used with little modifications (Tamura et al., 2009). Briefly, BMDMs $\left(1 \times 10^{6} / \mathrm{ml}\right)$ were pre-incubated with or without a hypotonic solution for $15 \mathrm{~min}$ and incubated with FITC-dextran $(1,000 \mu \mathrm{g} / \mathrm{ml})$ for $3 \mathrm{~h}$ at $37^{\circ} \mathrm{C}$ in normal environment. Cells were washed twice with PBS. Cells were collected and analyzed on BD LSR-II flow cytometer. The mean fluorescence intensity (MFI) of cells incubated with FITC-dextran at $0^{\circ} \mathrm{C}$ was set as fluorescence background. BMDMs which pretreated with isotonic solution served as control group.

To examine the phagocytosis, cells were treated in the same way with pinocytosis. After hypotonic stimulation, cells were cocultured with IgG-coated latex particle in 1:10 ratio for 5 min at $37^{\circ} \mathrm{C}$, and their phagocytosis was compared with the control group. The reaction was stopped by the addition of $2 \mathrm{ml}$ icecold PBS, and non-engulfed beads were removed with Accutase. Cells were washed four times with cold PBS and fixed in formaldehyde. Fifteen fields with phagocytosis were randomly chosen under light microscopy, and ingested beads of at least 200 macrophages were quantitated. Phagocytosis index (Link et al., 2010) was calculated by a fomula: Phagocytosis index = $(\Sigma n P n) / 1000$, from $n=0$ to $n=10$, where $n$ is the number of engulfed particles by a macrophage, and $\mathrm{Pn}$ is the percent of cells that phagocytosed $n$ particles.

\section{Expression of CD80, CD86, and MHC II Ag}

To check up the influence of hypotonicity on the antigen presenting capability of BMDMs, the expression of CD80, CD86 and MHC class II was measured by using flow cytometry.

BMDMs $\left(1 \times 10^{6} / \mathrm{ml}\right)$ were pre-incubated with or without the hypotonic solution for $15 \mathrm{~min}$, and cultured in normal environment for $3 \mathrm{~h}$. Cells $\left(1 \times 10^{6}\right)$ were stained with fluorescent mAbs diluted in PBS containing 1\% BSA (FACS buffer). Before staining, cells were incubated with purified anti-mouse CD16/32 for 10 min on ice to block FcR. The following Abs were used: FITC-labeled anti-CD80, PE-labeled anti-CD86, PE-cy5-labeled MHC class II, and APC-labeled F4/80. The cells were incubated for $30 \mathrm{~min}$ at room temperature, washed twice with FACS buffer and analyzed by LSR II flow cytometer (BD Bioscience, USA). The results were shown as mean fluorescence intensity (MFI). BMDMs which pretreated with isotonic solution served as control group.

\section{Cytokine production}

The effect of hypotonicity on IL-10 and TNF- $\alpha$ production was determined by using ELISA.

Briefly, BMDMs $\left(2 \times 10^{6} / \mathrm{ml}\right)$ were pre-incubated in the presence or absence of the hypotonic solution for $15 \mathrm{~min}$, then in isotonic solution for $6 \mathrm{~h}$. Supernatants were harvested for analyzing the levels of TNF- $\alpha$ and IL-10 by mouse ELISA kits. 100 $\mathrm{ng} / \mathrm{ml}$ LPS (sigma, USA) was used as a positive control. BMDMs which pretreated with isotonic solution served as control group.

\section{RT-PCR}

BMDMs were generated as previously described. After treated with hypotonicity, cells were used for the following test. Total RNA was isolated from BMDMs using TRIzol reagent according to the manufacturer's instructions. cDNA synthesis was performed using a PrimeScript first Strand cDNA Synthesis Kit. PCR amplifications of cDNA were performed by standard methods. The heart tissue obtained from the same mouse with BMDMs, and act as a positive control. The following specific mouse primers (forward and reverse) were used:

CIC-2 (Enz et al., 1999) 5'-CAA GTT CCT CTC CCT CTT G3', 5'-GAA CTG TCC AAA GCC AGG G-3'; CIC-3 (Okamoto et al., 2008), 5'-TGT GTC TCT GGT GGT TAT TG-3', 5'-GGA AGA GAT GGA GTA TGC TG-3'; GAPDH, 5'-AAC CTG CCA AGT ATG ATG AC-3', 5'-TAC CAG GAA ATG AGC TTG AC-3'. GAPDH was used for normalization. PCR products were analyzed by $2 \%$ agarose gel electrophoresis.

\section{Western blot}

After treated with hypotonicity, BMDMs were harvested as previously described and were used for the following test. BMDMs were lysed in lysis buffer (50 mM Tris [pH 8.0], $150 \mathrm{mM} \mathrm{NaCl}$ containing 1\% Nonidet P-40, 0.02\% NaN3, 0.5 mM PMSF, 1 
Hypotonicity Increased Macrophages Endocytosis via CIC-3

Yutao Yan et al.

A
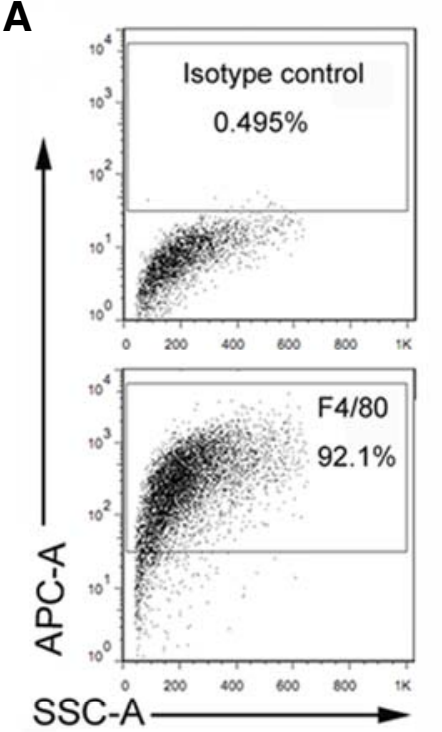

E
B

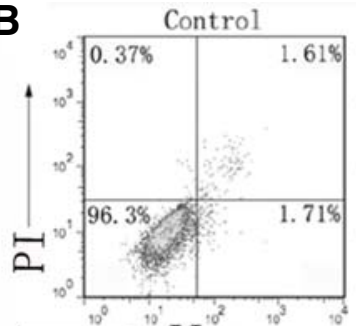

Annexin $\mathrm{V}^{10}$

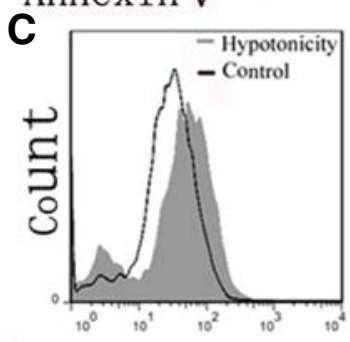

FITC-A
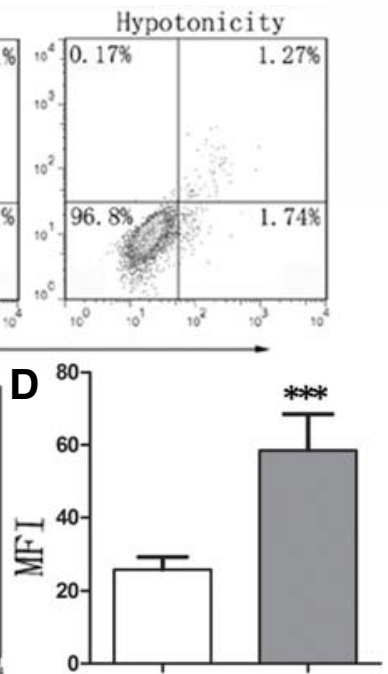

Control Hypotonicity

$\mathbf{F}$

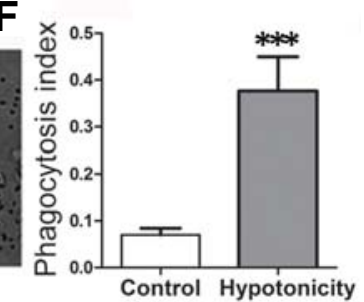

Fig. 1. Hypotonic environment promotes endocytosis in BMDMs. (A) The purity of BMDMs analyzed by flow cytometry. IgG was used as an isotype control. (B) Apoptotic fraction of cells detected by annexin $\vee$ staining ( $x$-axis)/propidium iodide staining (y-axis) after treatment with hypotonic solution. (C, D) Gray fill represents hypotonic group. (C) Pinocytotic activity of BMDMs in hypotonic environment shown by mean fluorescence intensity (MFI) of cells incubated with FITC-dextran. (E, F) Uptake of IgG-coated latex beads analyzed by light microscopy and calculated as phagocytosis index. Photos taken from a representative experiment. The data represent mean \pm SD from five independent experiments ( $\mathrm{n}=5$ for each group). ${ }^{\star \star \star} P<0.001$ for group comparisons.

A

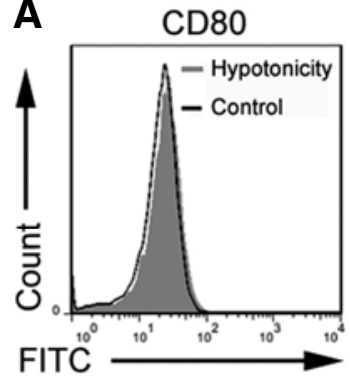

B

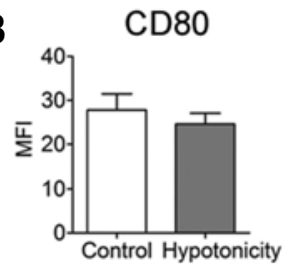

CD86
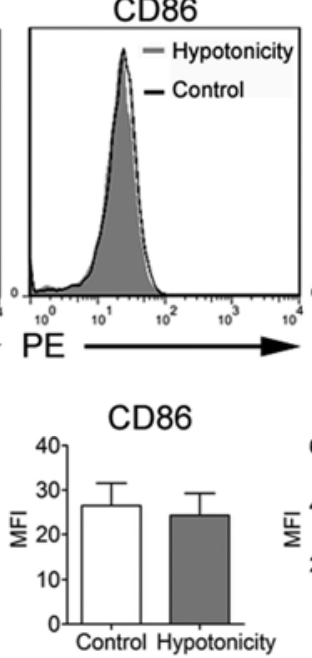

MHC class II
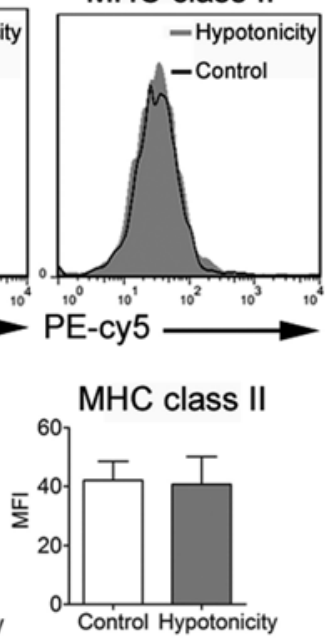

Fig. 2. Expression of CD80, CD86, and MHC class II molecules in BMDMs was not affected by hypotonic stress. (A) Histograms from representative experiments. Gray fill represents hypotonic group. (B) MFI values of CD80, CD86, and MHC class II analyzed by flow cytometry ( $n=5$ for each group).

$\mathrm{mg} / \mathrm{ml}$ aprotinin, and $1 \mathrm{mg} / \mathrm{ml}$ leupeptin). The whole-cell lysate was mixed with $5 \times$ SDS loading buffer and boiled for $10 \mathrm{~min}$. The proteins were separated by electrophoresis on $10 \%$ SDS- polyacrylamide gel and transferred to a polyvinylidene difluoride membrane. The membrane was blocked with $5 \%(\mathrm{w} / \mathrm{v})$ nonfat milk in TBST for $2 \mathrm{~h}$ at $37^{\circ} \mathrm{C}$ and then incubated with rabbit 


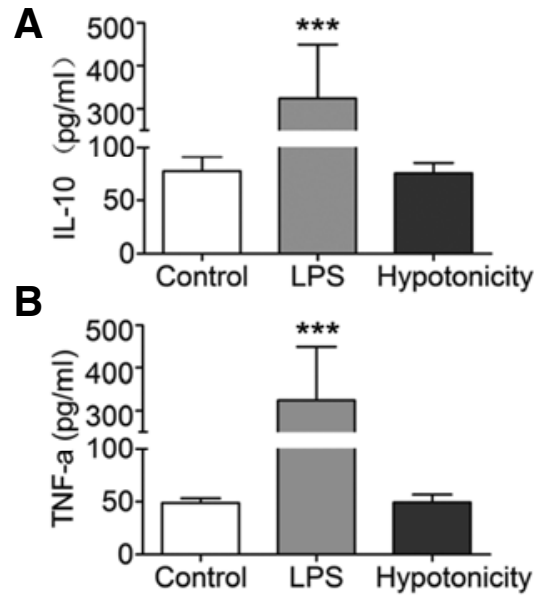

Fig. 3. Secretion of TNF- $\alpha$ or IL-10 by BMDMs was not affected by hypotonic stress. (A, B) Cytokine levels quantified by ELISA. LPS group served as positive control. ( $\mathrm{n}=3$ for earch group). ${ }^{\star \star \star} P<$ 0.001 for LPS group compared to control and hypotonicity groups.

anti-ClC-3 or rabbit anti-ClC-2 Abs, as well as mouse antiGAPDH antibody (serving as a loading control) diluted in 5\% $(\mathrm{w} / \mathrm{v})$ nonfat milk in $0.1 \%$ TBST overnight at $4^{\circ} \mathrm{C}$. After washing three times (10 min each) in 0.1\% TBST, the membranes were incubated with peroxidase-conjugated goat anti-rabbit secondary antibody for $2 \mathrm{~h}$ at $37^{\circ} \mathrm{C}$. The membrane was washed three times in $0.1 \%$ TBST, and the proteins were detected using Peroxide Solution with a Kodak Image Station 4000MM.

For negative controls, the specificity of anti-CIC-3 antibody $(\mathrm{ClCn} 3)$ was confirmed by incubating the membrane fraction with the antigen pre-absorbed antibody. The antigen of CIC-3 with a molecular weight of $35 \mathrm{kDa}$ was provided by Alomone labs.

\section{Immunocytochemistry}

The immunofluorescence was used to determine the localization of CIC-3 in BMDMs. First, BMDMs were cultured on glass coverslips, washed with $\mathrm{PBS}$ once, fixed with $4 \%$ paraformaldehyde in PBS for 20 min, washed three times in PBS, permeabilized with $0.3 \%$ Triton X-100 in PBS for 20 min, washed three times for $5 \mathrm{~min}$ in PBS, and then blocked with 3\% BSA for $2 \mathrm{~h}$ at $37^{\circ} \mathrm{C}$. BMDMs were incubated with rabbit anti-CIC-3 primary Abs diluted in $3 \% \mathrm{BSA}$ overnight at $4^{\circ} \mathrm{C}$. After washing three times in PBS, cells were incubated with the FITCconjugated goat anti-rabbit secondary antibody diluted in $3 \%$ BSA for $1 \mathrm{~h}$ at room temperature. After extensive washing, coverslips were mounted on glass slides with $50 \%$ glycerol. Staining was visualized with an Olympus FV500 confocal microscope.

\section{siRNA-mediated knockdown of CIC-3}

The 21-nucleotide siRNA(Okamoto et al., 2008) duplexes with two overhang dT nucleotides at the 3-end targeted to the mouse CIC-3 channel were designed and synthesized by Invitrogen (USA). The following specific primers (forward and reverse) were used: CIC-3, siRNA 5'-CGA GAG AAG UGU AAG GAC ATT-3', 5'-UGU CCU UAC ACU UCU CUC GTT-3'; control siRNA, 5'-AUC CGC GCG AUA GUA CGU ATT-3', 5'-UAC GUA CUA UCG CGC GGA UTT-3'. siRNAs were transfected with nuclear transfer according to the manufacturer's instruction.
BMDMs were harvested and transfected with FAM (carboxyfluorescein)-conjugated CIC-3 siRNA. 6 or $72 \mathrm{~h}$ after transfection, BMDMs were collected to test transfection efficiency by flow cytometry and interference efficiency by Western blot. After transfection, BMDMs were stimulated with hypotonic and the endocytosis activity was measured. BMDMs without transfection served as normal control group, and transfected with control siRNA served as control siRNA group.

\section{Statistical analysis}

Data are presented as mean \pm SD. The Student's $t$-test with paired comparisons was used to evaluate the differences. $P<$ 0.05 was considered statistically significant.

\section{RESULTS}

Purity of BMDMs

To insure sufficient purity of BMDMs, the cells were tested by flow cytometry and achieved a purity of $92.1 \%$ (Fig. 1A).

Effect of hypotonic solution on apoptosis of BMDMs To determine if a hypotonic solution can affect the function of BMDMs and induce apoptosis, the Annexin V-FITC/PI double staining assay was used to detect apoptotic cells. The distribution of early apoptotic cells did not appreciably differ between BMDMs treated with an isotonic v. hypotonic solution $(1.71 \% \mathrm{v}$. $1.74 \%$, respectively; Fig. 1B).

Hypotonic environment promotes endocytosis by BMDMs Endocytosis is an important function of macrophages. To investigate the effect of a hypotonic environment on endocytic function in BMDMs, we examined pinocytosis of FITC-dextran and phagocytosis of IgG-coated latex beads. Both FITC-dextran pinocytosis (Figs. 1C and 1D) and IgG-coated latex phagocytosis (Figs. $1 \mathrm{E}$ and $1 \mathrm{~F})$ were significantly increased $(P<0.001)$ after stimulation of BMDMs with a hypotonic solution compared to BMDMs in a control isotonic environment (arrows show ingested particles/beads).

Effect of hypotonic environment on expression of CD80, CD86, and MHC class II molecules in BMDMs

Macrophages act as APCs to trigger adaptive immunity. To further examine the effect of a hypotonic environment on the APC function of macrophages, expression of CD80, CD86 and $\mathrm{MHC}$ class II molecules in BMDMs was examined by flow cytometry. As shown in Figs. $2 \mathrm{~A}$ and $2 \mathrm{~B}$, there were no obvious differences between the hypotonic and control groups.

Effect of hypotonic environment on secretion of IL-10 and TNF- $\alpha$ by BMDMs

IL-10 and TNF- $\alpha$ are anti- and pro-inflammatory cytokines, respectively, released by activated BMDMs. To examine the effect of a hypotonic environment on secretion of these cytokines, IL-10 and TNF- $\alpha$ were quantified by ELISA. LPS was used as positive control. As shown in Figs. $3 \mathrm{~A}$ and $3 \mathrm{~B}$, following stimulation of BMDMs by LPS, levels of both IL-10 and TNF- $\alpha$ were significantly increased in BMDM culture supernatants $(P<$ 0.001 ), whereas levels of either cytokine were unchanged when BMDMs were placed in a hypotonic environment.

\section{Expression of CIC-3 in BMDMs}

It has been previously reported that $\mathrm{CIC}-2$ and $\mathrm{CIC}-3$, expressed in diverse tissues including heart (Dick et al., 1999), mediate changes in cellular function in a hypotonic environment. 
A

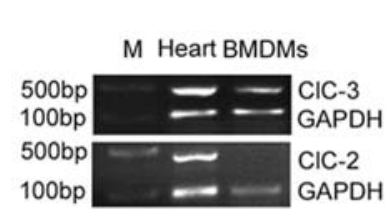

C

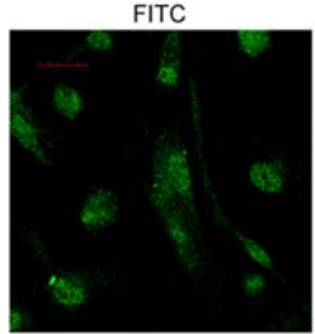

D
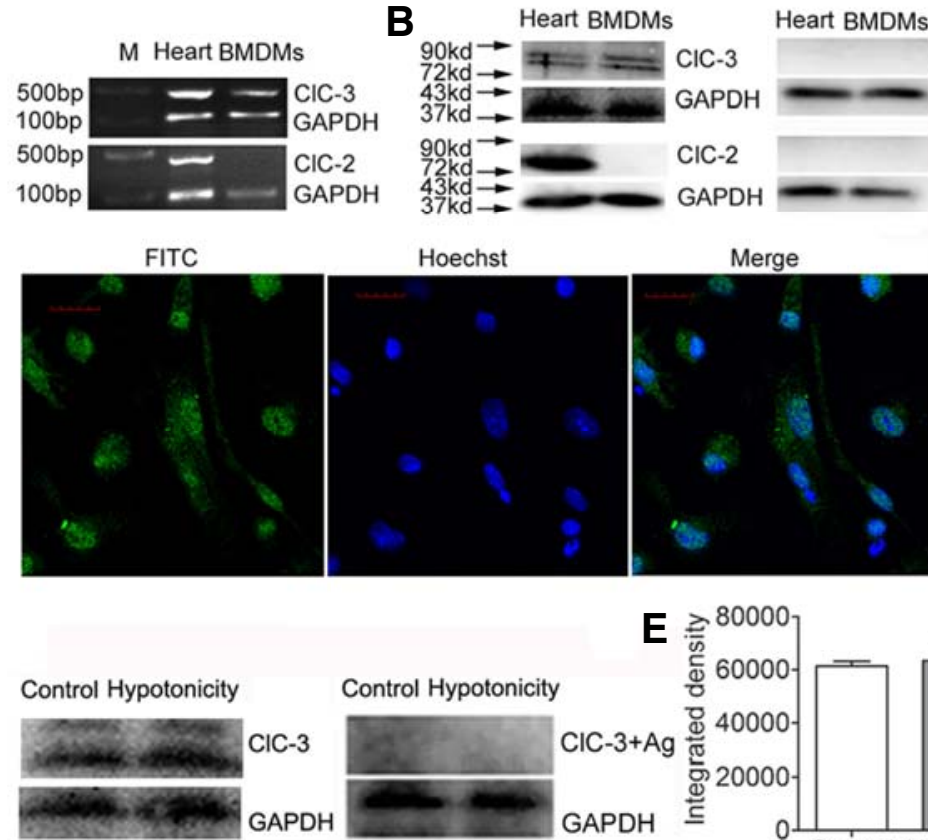

$\mathrm{ClC}-3+\mathrm{Ag}$

GAPDH

$\mathrm{CIC}-2+\mathrm{Ag}$ GAPDH
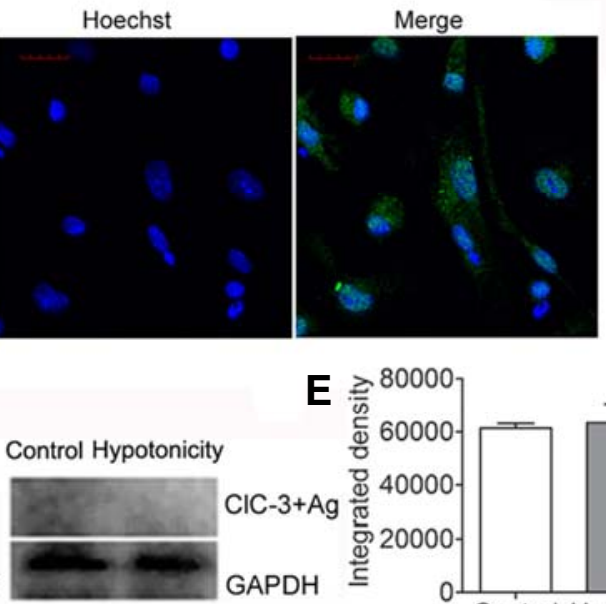

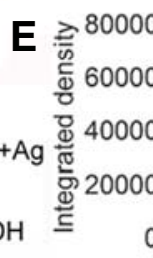

$\mathbf{F}$

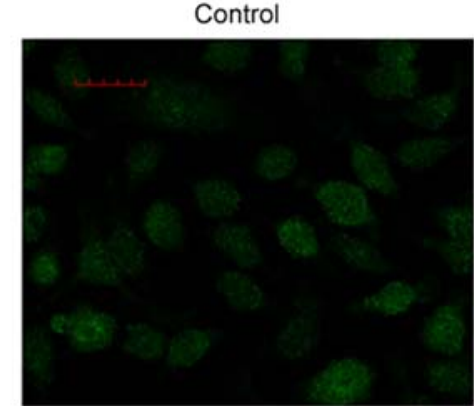

Hypotonicity

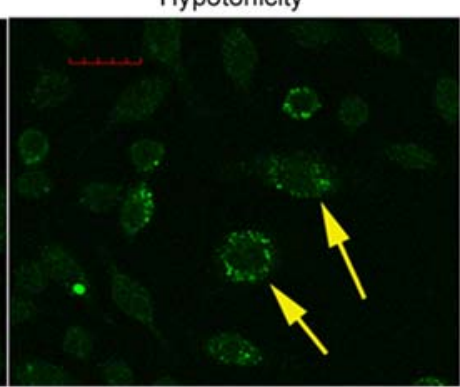

Fig. 4. Expression and localization of $\mathrm{ClC}-2$ and $\mathrm{ClC}-3$ in BMDMs. (A) CIC transcripts detected in BMDMs by RTPCR. Heart tissue was used as positive control. (B) ClC protein expression in BMDMs measured by Western blot. Heart tissue served as positive control. For specific controls, antibodies against $\mathrm{CICs}$ were pre-incubated with corresponding peptide antigens. (C) Localization of CIC-3 in BMDMs shown by double-staining immunofluorescence (original magnification $\times 1000)$. Scale bar $=$ $20 \mu \mathrm{m}$. Nuclei (blue) and CIC-3 (Green) were counterstained with Hoechst 33342 and $\mathrm{CIC}-3$ antibody, respectively. (D, E) CIC-3 expression in BMDMs after hypotonic exposure examined by Western blot. Photos taken from a representative experiment. The data represent mean \pm SD from three independent experiments. (F) Localization of CIC-3 in BMDMs stimulated with hypotonic solution visualized by double-staining immunofluorescence (original magnification $\times 1000$ ). Scale bar $=20 \mu \mathrm{m}$. ClC-3 collected in clusters around the cytomembrane, shown by arrows ( $\mathrm{n}=3$ for each group).
The expression of $\mathrm{ClC}-3$ and $\mathrm{CIC}-2$ mRNA in BMDMs was examined using RT-PCR. The sizes of the expected PCR products for CIC-3 and CIC-2 were 456 and 499 bp, respectively. We found that only CIC-3 mRNA was expressed in BMDMs, where as both transcripts were expressed in heart (Fig. 4A). The expression of $\mathrm{CIC}-3$ and $\mathrm{CIC}-2$ proteins in BMDMs was detected using Western blot and immunocytochemistry. As shown in Fig. 4B, CIC-3 but not CIC-2 was detected in BMDMs. CIC-3 resolved as two bands between 72 and $90 \mathrm{kDa}$ on Western blot, which may reflect glycosylated and nonglycosylated isoforms, and was predominantly expressed in the cytoplasm of BMDMs determined by immunocytochemistry (Fig. 4C). Stimulation of BMDMs by a hypotonic solution did not alter CIC-3 expression, but distribution of the protein changed to accumulate in clusters around the cytomembrane (shown by arrows, Fig. 4F right panel).

Increased endocytosis in BMDMs under hypotonic environment via $\mathrm{CIC}-3$

To investigate if $\mathrm{CIC}-3$ is required for the increased endocytic activity of BMDMs under hypotonic conditions, siRNA was used to suppress the expression of $\mathrm{ClC}-3$. Flow cytometric analysis showed that the siRNA transfection efficiency of control and CIC-3 siRNAs tracked by FAM was $42.6 \%$ and $46.4 \%$, respec- tively (Fig. 5A), while the interference efficiency of CIC-3 siRNA was approximately $50 \%$ (Figs. $5 \mathrm{~B}$ and $5 \mathrm{C}$ ). Uptake of both FITC-dextran (Fig. 5D) and IgG-coated latex beads (Figs. 5E and $5 \mathrm{~F}$ ) in hypotonia-exposed BMDMs was significantly decreased in the group transfected with $\mathrm{CIC}-3$ siRNA compared to the normal control and control siRNA groups $(P<0.001)$.

\section{DISCUSSION}

Macrophages play an important role in innate immunity and adaptive immune responses, including destruction of pathogens, endocytosis of antigens, and possible mediation of tumor development (Torr et al., 2012). Endocytosis is an important macrophage function. Studies to date have shown that endocytosis in macrophages is regulated by many factors, including cytokines (Parveen et al., 2013; Roger et al., 2013), medicines (Li and Liu, 2005) and extracellular acidosis (Kong et al., 2013). Utilizing endocytosis, macrophages can eliminate invading micro-organisms and remove necrotic tissue and cells. Our results suggest that hypotonic stress may enhance the endocytic activity of macrophages.

In the present study, we examined the impact of extracellular hypotonicity on BMDMs and investigated the mechanism by which hypotonic stress regulates their function. First, we 
A

B

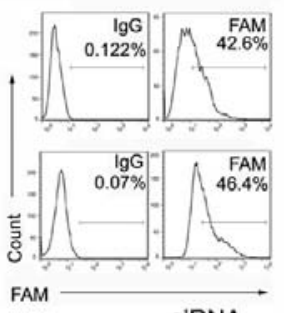

D

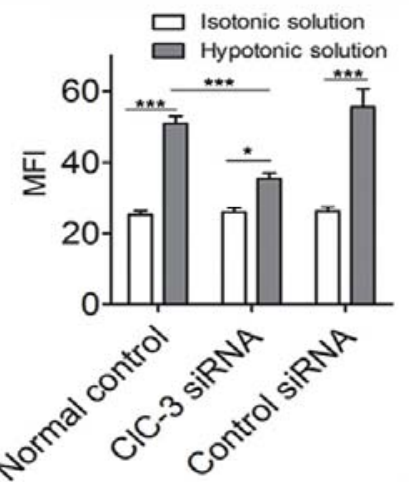

C

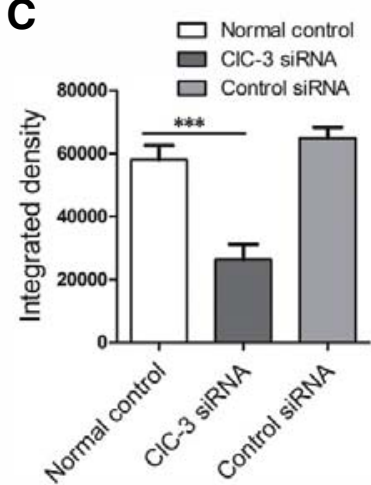

E

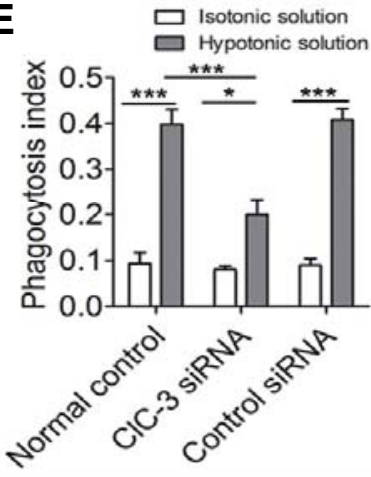

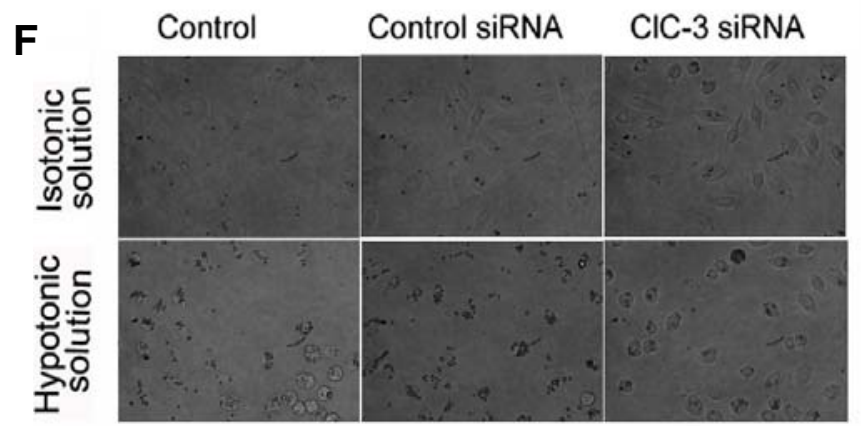

examined the effect of hypotonic stress on endocytosis in BMDMs. Recent work has shown that during ischemiareperfusion episodes, the local tissue microenvironment becomes hypotonic, producing additional tissue damage and cell death (Okada et al., 2004; 2009). Macrophages are known to play a role in the immunopathology of ischemia-reperfusion injury (Zhang et al., 2013), but the extent to which hypotonic stress affects macrophage function is unknown. In our experiments, FITC-dextran was used to detect pinocytosis, while IgGcoated latex beads were used to monitor receptor-mediated endocytosis in BMDMs. Our results showed that extracellular hypotonic stress increased both pinocytosis and receptormediated phagocytosis in BMDMs.

CD80, CD86 and MHC-II molecules are known markers of macrophage maturation. To examine the effect of hypotonic stress on BMDM maturation, we quantified expression of these molecules during hypotonic stress. After the exposure of BMDMs to hypotonicity, levels of CD80, CD86 and MHC-II secreted into BMDM cell culture supernatants were similar in the hypotonic and control groups. Activated macrophages also

Fig. 5. ClC-3 plays a role in endocytic activity of BMDMs under hypotonic stress. (A) The efficiency of CIC-3 siRNA transfection measured by flow cytometry. IgG was used for isotype control. (B, C) Protein expression of $\mathrm{ClC}-3$ after transfection detected by Western blot. (D) MFI values for normal control, CIC-3 siRNA and control siRNA groups analyzed by flow cytometry. Pinocytotic activity in BMDMs was decreased by interference with CIC-3. (E, F) Uptake of IgG-coated latex analyzed as phagocytosis index in normal control, CIC-3 siRNA and control siRNA groups. Phagocytic activity in BMDMs was down-regulated by interference with CIC-3. Photos taken from a representative experiment. The data represent mean \pm SD from three independent experiments ( $\mathrm{n}=3$ for each group). ${ }^{\star} P<0.05$, ${ }^{\star \star \star} P<0.001$ for differences between treatment groups. secrete different cytokines, two of which, IL-10 and TNF- $\alpha$, are inhibitory and stimulatory cytokines, respectively. Our results showed that after exposure to hypotonic stress, IL-10 and TNF$\alpha$ levels in BMDM culture supernatants were unchanged.

The mechanism by which BMDMs sense extracellular hypotonic stress is unclear. Recent work has focused on the role of ion conduction pathways in lymphocyte function and immunity. Our group has shown that acid sensing ion channels are crucially involved in the modulation of BMDMs and contribute to the effect acidosis imposes on dendritic cells (Kong et al., 2013; Tong et al., 2011).

In our study, we examined ion channels $\mathrm{CIC}-2$ and $\mathrm{ClC}-3$, members of the chloride channel superfamily. These channels play important roles in the regulation of cellular excitability, cell volume regulation, and acidification of intracellular organelles (Tang and Chen, 2011). Previous studies have reported that CIC-2 and CIC-3 mediate functional changes induced by hypotonicity. For example, CIC-2 was shown to control the functional response of trabecular meshwork cells to a hypotonic environment (Comes et al., 2005). A more recent study using an induc- 
ible heart-specific CIC-3 knockout mouse found that inactivation of CIC-3 gene produced myocardial hypertrophy and heart failure (Xiong et al., 2010). In our study, we confirmed the expression of ClC-3 in BMDMs by RT-PCR, Western blot and immunocytochemistry. We used the $\mathrm{Cl}^{-}$channel blocker 5-nitro2-(3-phenylpropylamino) benzoic acid (data not shown) and CIC-3 siRNA to investigate the role of $\mathrm{CIC}-3$ in mediating the effects of extracellular hypotonicity on the biological behavior of BMDMs. We found that CIC-3 siRNA abrogated the increase in endocytosis induced by hypotonic stress. These data provide strong evidence that $\mathrm{CIC}-3$ is crucially involved in stimulating endocytic activity in BMDMs by hypotonic stress.

Our results also showed that stimulation of BMDMs by a hypotonic solution had no significant impact on $\mathrm{CIC}-3$ expression but rather altered distribution of the protein into clusters of accumulation around the cytomembrane. Two explanations may account for this phenomenon. On the one hand, our results were similar to findings in neurons and mouse osteoclasts where $\mathrm{CIC}-3$ expression predominantly localized to endosomal compartments in the cytoplasm (Stobrawa et al., 2001). We propose that in BMDMs CIC-3 may be located to lysosomes where it functions in lysosome acidification. The exchanger may produce a $\mathrm{Cl}^{-}$current into a subcellular vesicle, reducing the potential difference caused by electrogenic $\mathrm{H}^{+}$transport. On the other hand, CIC-3 is an important component of volumesensitive outwardly rectifying chloride channels (VSORs) (Hermoso et al., 2002). VSORs are ubiquitously expressed and involved in cell volume regulation after osmotic swelling, called regulatory volume decrease, in various cell types (Inoue et al., 2010; Min et al., 2011). The hypotonicity-induced relocation of CIC-3 we observed in BMDMs may be related to endocytosis. We hypothesize that intracellular CIC-3 may act as a cell volume sensor and translocate to the cell membrane in response to cellular swelling induced by hypotonic stress. Consequently, VSORs become activated and redundant materials are discharged from the BMDMs in order to increase macrophage endocytic capacity and efficiency.

To exclude the possibility that apoptosis in BMDMs induced by hypotonic stress resulted in increased endocytic activity, we carried out flow cytometric apoptosis detection. Our results showed that apoptosis was nearly undetectable in BMDMs treated with the hypotonic solution used in our study. The endocytic activity of BMDMs induced by hypotonic stress thus appears to have no relationship to apoptosis.

Recently, studies have shown that $\mathrm{ClC}-3$ is necessary for activation of smooth muscle cells by TNF- $\alpha$. Deficiency in CIC-3 markedly reduced neointimal hyperplasia following vascular injury (Chu et al., 2011). Our investigation showed that CIC-3 is also involved in macrophage function. Control of CIC-3 may represent a novel therapeutic target to prevent clinical blood vessel damage. Furthermore, intraperitoneal chemotherapy with hypotonic perfusion is an important technique for the prevention of postoperative retroperitoneal tumor relapse. The mechanism underlying this therapy is thought to involve increased permeability of the tumor cell membrane in response to hypotonicity, rendering tumor cells more sensitive to chemotherapy and thus more easily killed. Our study suggests a new pharmacodynamic mechanism in which the endocytic ability of macrophages is enhanced by a hypotonic environment, allowing macrophages to engulf tumor cells and debris more efficiently.

In summary, the increased endocytic ability of BMDMs in a hypotonic environment appears to require $\mathrm{CIC}-3$, which may depend on VSORs and organelle acidification.

\section{ACKNOWLEDGMENTS}

This work was supported by grants from the National Natural Science Foundation of China (No. 81273221) and Major State Basic Research Development Program of China (973 Program) (No. 2013CB530505).

\section{REFERENCES}

Abdullaev, I.F., Sabirov, R.Z., and Okada, Y. (2003). Upregulation of swelling-activated $\mathrm{Cl}$ - channel sensitivity to cell volume by activation of EGF receptors in murine mammary cells. J. Physiol. $549,749-758$

Bozza, M.T., Martins, Y.C., Carneiro, L.A., and Paiva, C.N. (2012) Macrophage migration inhibitory factor in protozoan infections. J. Parasitol. Res. 2012, 413052.

Cassetta, L., Cassol, E., and Poli, G. (2011). Macrophage polarization in health and disease. ScientificWorldJournal 11, 2391-2402.

Chu, X., Filali, M., Stanic, B., Takapoo, M., Sheehan, A., Bhalla, R., Lamb, F.S., and Miller, F.J., Jr. (2011). A critical role for chloride channel-3 (CIC-3) in smooth muscle cell activation and neointima formation. Arterioscler. Thromb. Vasc. Biol. 31, 345351.

Comes, N., Gasull, X., Gual, A., and Borras, T. (2005). Differential expression of the human chloride channel genes in the trabecular meshwork under stress conditions. Exp. Eye Res. 80, 801-813.

Dick, G.M., Kong, I.D., and Sanders, K.M. (1999). Effects of anion channel antagonists in canine colonic myocytes: comparative pharmacology of $\mathrm{Cl}^{-}, \mathrm{Ca}^{2+}$ and $\mathrm{K}^{+}$currents. $\mathrm{Br}$. J. Pharmacol. 127, 1819-1831.

Dickerson, R.N., Maish, G.O., 3rd, Weinberg, J.A., Croce, M.A Minard, G., and Brown, R.O. (2013). Safety and efficacy of intravenous hypotonic $0.225 \%$ sodium chloride infusion for the treatment of hypernatremia in critically III patients. Nutr. Clin. Pract. 28, 400-408.

Duan, D.D. (2011). The CIC-3 chloride channels in cardiovascular disease. Acta Pharmacol. Sin. 32, 675-684.

Enz, R., Ross, B.J., and Cutting, G.R. (1999). Expression of the voltage-gated chloride channel CIC-2 in rod bipolar cells of the rat retina. J. Neurosci. 19, 9841-9847.

Gaglio, P., Marfo, K., and Chiodo, J., 3rd (2012). Hyponatremia in cirrhosis and end-stage liver disease: treatment with the vasopressin V(2)-receptor antagonist tolvaptan. Dig. Dis. Sci. 57, 2774-2785.

Gong, D., Shi, W., Yi, S.J., Chen, H., Groffen, J., and Heisterkamp, N. (2012). TGFbeta signaling plays a critical role in promoting alternative macrophage activation. BMC Immunol. 13, 31.

Hermoso, M., Satterwhite, C.M., Andrade, Y.N., Hidalgo, J., Wilson, S.M., Horowitz, B., and Hume, J.R. (2002). CIC-3 is a fundamental molecular component of volume-sensitive outwardly rectifying $\mathrm{Cl}$ - channels and volume regulation in $\mathrm{HeLa}$ cells and Xenopus laevis oocytes. J. Biol. Chem. 277, 4006640074.

Inoue, H., Takahashi, N., Okada, Y., and Konishi, M. (2010). Volume-sensitive outwardly rectifying chloride channel in white adipocytes from normal and diabetic mice. Am. J. Physiol. Cell Physiol. 298, C900-909.

Kajimoto, K., Shao, D., Takagi, H., Maceri, G., Zablocki, D., Mukai, H., Ono, Y., and Sadoshima, J. (2011). Hypotonic swellinginduced activation of PKN1 mediates cell survival in cardiac myocytes. Am. J. Physiol. Heart Circ. Physiol. 300, H191-200.

Kondo, A., Maeta, M., Oka, A., Tsujitani, S., Ikeguchi, M., and Kaibara, N. (1996). Hypotonic intraperitoneal cisplatin chemotherapy for peritoneal carcinomatosis in mice. Br. J. Cancer 73, 1166-1170.

Kong, X., Tang, X., Du, W., Tong, J., Yan, Y., Zheng, F., Fang, M., Gong, F., and Tan, Z. (2013). Extracellular acidosis modulates the endocytosis and maturation of macrophages. Cell. Immunol. $281,44-50$

$\mathrm{Li}, \mathrm{X}$., and Liu, X. (2005). Effect of curcumin on immune function of mice. J. Huazhong Univ. Sci. Technol. Med. Sci. 25, 137-140.

Link, T.M., Park, U., Vonakis, B.M., Raben, D.M., Soloski, M.J., and Caterina, M.J. (2010). TRPV2 has a pivotal role in macrophage 
particle binding and phagocytosis. Nat. Immunol. 11, 232-239.

Liu, X., Silverstein, P.S., Singh, V., Shah, A., Qureshi, N., and Kumar, A. (2012). Methamphetamine increases LPS-mediated expression of IL-8, TNF- $\alpha$ and IL-1 $\beta$ in human macrophages through common signaling pathways. PLoS One 7, e33822.

Medina-Contreras, O., Geem, D., Laur, O., Williams, I.R., Lira, S.A., Nusrat, A., Parkos, C.A., and Denning, T.L. (2011). CX3CR1 regulates intestinal macrophage homeostasis, bacterial translocation, and colitogenic Th17 responses in mice. J. Clin. Invest. 121, 4787-4795.

Min, X.J., Li, H., Hou, S.C., He, W., Liu, J., Hu, B., and Wang, J. (2011). Dysfunction of volume-sensitive chloride channels contributes to cisplatin resistance in human lung adenocarcinoma cells. Exp. Biol. Med. 236, 483-491.

Mohammad-Panah, R., Harrison, R., Dhani, S., Ackerley, C., Huan, L.J., Wang, Y., and Bear, C.E. (2003). The chloride channel $\mathrm{CIC}-4$ contributes to endosomal acidification and trafficking. J. Biol. Chem. 278, 29267-29277.

Okada, Y., Maeno, E., Shimizu, T., Manabe, K., Mori, S., and Nabekura, T. (2004). Dual roles of plasmalemmal chloride channels in induction of cell death. Pflugers Arch. 448, 287-295.

Okada, Y., Sato, K., and Numata, T. (2009). Pathophysiology and puzzles of the volume-sensitive outwardly rectifying anion channel. J. Physiol. 587, 2141-2149.

Okamoto, F., Kajiya, H., Toh, K., Uchida, S., Yoshikawa, M., Sasaki, S., Kido, M.A., Tanaka, T., and Okabe, K. (2008). Intracellular $\mathrm{ClC}-3$ chloride channels promote bone resorption in vitro through organelle acidification in mouse osteoclasts. Am. J. Physiol. Cell Physiol. 294, C693-701.

Park, K.R., and Bryers, J.D. (2012). Effect of macrophage classical (M1) activation on implant-adherent macrophage interactions with Staphylococcus epidermidis: a murine in vitro model system. J. Biomed. Mater. Res. A 100, 2045-2053.

Parveen, N., Varman, R., Nair, S., Das, G., Ghosh, S., and Mukhopadhyay, S. (2013). Endocytosis of Mycobacterium tuberculosis heat shock protein 60 is required to induce production in macrophages. J. Biol. Chem. 288, 24956-24971.

Roberts, B.N., and Christini, D.J. (2011). NHE inhibition does not improve $\mathrm{Na}(+)$ or $\mathrm{Ca}(2+)$ overload during reperfusion: using modeling to illuminate the mechanisms underlying a therapeutic failure. PLoS Comput. Biol. 7, e1002241.

Roger, T., Delaloye, J., Chanson, A.L., Giddey, M., Le Roy, D., and Calandra, T. (2013). Macrophage migration inhibitory factor deficiency is associated with impaired killing of gram-negative bacteria by macrophages and increased susceptibility to Klebsiella pneumoniae sepsis. J. Infect. Dis. 207, 331-339.

Schliess, F., Foster, N., Gorg, B., Reinehr, R., and Haussinger, D. (2004). Hypoosmotic swelling increases protein tyrosine nitration in cultured rat astrocytes. Glia 47, 21-29.
Shapiro, H., Lutaty, A., and Ariel, A. (2011). Macrophages, metainflammation, and immuno-metabolism. ScientificWorldJournal $11,2509-2529$

Siegel, A.J. (2007). Hypertonic (3\%) sodium chloride for emergent treatment of exercise-associated hypotonic encephalopathy. Sports Med. 37, 459-462.

Stobrawa, S.M., Breiderhoff, T., Takamori, S., Engel, D., Schweizer, M., Zdebik, A.A., Bosl, M.R., Ruether, K., Jahn, H., Draguhn, A., et al. (2001). Disruption of ClC-3, a chloride channel expressed on synaptic vesicles, leads to a loss of the hippocampus. Neuron 29, 185-196.

Tamura, N., Hazeki, K., Okazaki, N., Kametani, Y., Murakami, H., Takaba, Y., Ishikawa, Y., Nigorikawa, K., and Hazeki, O. (2009). Specific role of phosphoinositide 3-kinase p110alpha in the regulation of phagocytosis and pinocytosis in macrophages. Biochem J. 423, 99-108.

Tang, C.Y., and Chen, T.Y. (2011). Physiology and pathophysiology of CIC-1: mechanisms of a chloride channel disease, myotonia. J. Biomed. Biotechnol. 2011, 685328

Thorp, E.B. (2012). Contrasting inflammation resolution during atherosclerosis and post myocardial infarction at the level of monocyte/macrophage phagocytic clearance. Front. Immunol. 3, 39.

Tong, J., Wu, W.N., Kong, X., Wu, P.F., Tian, L., Du, W., Fang, M. Zheng, F., Chen, J.G., Tan, Z., et al. (2011). Acid-sensing ion channels contribute to the effect of acidosis on the function of dendritic cells. J. Immunol. 186, 3686-3692.

Torr, E.E., Gardner, D.H., Thomas, L., Goodall, D.M., Bielemeier, A., Willetts, R., Griffiths, H.R., Marshall, L.J., and Devitt, A. (2012). Apoptotic cell-derived ICAM-3 promotes both macrophage chemoattraction to and tethering of apoptotic cells. Cell Death Differ. 19, 671-679.

Tunkel, A.R., and Scheld, W.M. (1993). Pathogenesis and pathophysiology of bacterial meningitis. Ann. Rev. Med. 44, 103120.

Wheeler, T.M., Lueck, J.D., Swanson, M.S., Dirksen, R.T., and Thornton, C.A. (2007). Correction of CIC-1 splicing eliminates chloride channelopathy and myotonia in mouse models of myotonic dystrophy. J. Clin. Invest. 117, 3952-3957.

Xiong, D., Heyman, N.S., Airey, J., Zhang, M., Singer, C.A., Rawat, S., Ye, L., Evans, R., Burkin, D.J., Tian, H., et al. (2010). Cardiac-specific, inducible $\mathrm{CIC}-3$ gene deletion eliminates native volume-sensitive chloride channels and produces myocardial hypertrophy in adult mice. J. Mol. Cell. Cardiol. 48, 211-219.

Zhang, Y.X., Zhang, J.R., and Wang, Z.G. (2013). Mycophenolate mofetil affects monocyte Toll-like receptor 4 signaling during mouse renal ischemia/reperfusion injury. Chin. Med. J. 126, 1224-1229. 